\title{
CENTRALIZING PROPERTIES IN SIMPLE LOCALLY FINITE GROUPS AND LARGE FINITE CLASSICAL GROUPS
}

\author{
BRIAN HARTLEY
}

(Received 10 July 1989)

\author{
Communicated by $\mathrm{H}$. Lausch
}

Dedicatad to G. E. (Tim) Wall, in recognition of his distinguished contribution to mathematics in Australia, on the occasion of his retirement

\begin{abstract}
The following question is discussed and evidence for and against it is advanced: is it true that if $F$ is an arbitrary finite subgroup of an arbitrary non-linear simple locally finite group $G$, then $C_{G}(F)$ is infinite? The following points to an affirmative answer.

THEOREM A. Let $F$ be an arbitrary finite subgroup of a non-linear simple locally finite group $G$. Then there exist subgroups $D \triangleleft C \leq G$ such that $F$ centralizes $C / D, F \cap C \leq D$, and $C / D$ is a direct product of finite alternating groups of unbounded orders. In particular, $F$ centralizes an infinite section of $G$.

Theorem $A$ is deduced from a "local" version, namely

THEOREM B. There exists an integer valued function $f(n, r)$ with the following properties. Let $H$ be a finite group of order at most $n$, and suppose that $H \leq S$, where $S$ is either an alternating group of degree at least $f=f(n, r)$ or a finite simple classical group whose natural projective representation has degree at least $f$. Then there exist subgroups $D \triangleleft C \leq S$ such that (i) $[H, C] \leq D$, (ii) $H \cap C \leq D$, (iii) $C / D \cong \operatorname{Alt}(r)$, (iv) $D=1$ if $S$ is alternating, and $D$ is a p-group of class at most 2 and exponent dividing $p^{2}$ if $S$ is a classical group over a field of characteristic $p$.

The natural "local version" of our main question is however definitely false.

Proposition C. Let $p$ be a given prime. Then there exists a finite group $H$ that can be embedded in infinitely many groups $\operatorname{PSL}(n, p)$ as a subgroup with trivial centralizer.
\end{abstract}

1980 Mathematics subject classification (Amer. Math. Soc.) (1985 Revision): 20 F 50, 20 D 08.

(C) 1990 Australian Mathematical Society 0263-6115/90\$A2.00+0.00 


\section{Introduction}

The study of simple locally finite groups depends heavily on knowledge about finite simple groups, and, not surprisingly, the classification of finite simple groups has led to considerable progress in our understanding of simple locally finite groups, or $\mathfrak{S}$-groups, as we shall call them. In [6], we used the classification to discuss centralizers of elements in infinite $\mathfrak{S}$-groups, and in this paper we study centralizing properties of arbitrary finite subgroups of infinite $\mathfrak{S}$-groups. Now $\mathfrak{S}$-groups can be divided into two classes with widely differing properties, the linear ones and the non-linear ones. The infinite linear 5 -groups are precisely the Chevalley groups and their twisted analogues over infinite locally finite fields [1], [2], [7], [15]. They are known, although perhaps not fully understood, and powerful techniques are available for studying them. Among the non-linear ones, the finitary linear groups have recently received attention. These act faithfully on a vector space $V$ in such a way that for each element $x$, the kernel of $x-1$ has finite codimension. Although these form a very natural class and much is known about them [5], [13], we shall not distinguish them from other non-linear 5 -groups in this paper.

The following question, which we shall discuss but not answer, focuses attention on the issues we deal with in this paper.

QUESTION. Is it the case that in a non-linear $\mathfrak{G}$-group, the centralizer of every finite subgroup is infinite?

One could go further and ask whether the centralizer of every finite subgroup involves a non-linear $\mathfrak{G}$-group. It is known [6] that the centralizer of each element of an infinite $\mathfrak{G}$-group is infinite, irrespective of linearity, but any linear $\mathfrak{G}$-group will certainly contain finite subgroups with trivial centralizer. Further, [6, Theorem B] provides quite strong evidence to support the view that in a non-linear $\mathfrak{G}$-group, the centralizer of every element involves a non-linear $\mathfrak{G}$-group.

We shall prove the following.

THEOREM A. Let $G$ be any non-linear $\mathfrak{G}$-group and $F$ be any finite subgroup of $G$. Then there exist subgroups $D \triangleleft C \leq G$ such that $D$ contains $[C, F]$ and $C \cap F$, and $C / D$ is a direct product of finite alternating groups of unbounded orders.

Although at first sight this seems like substantial progress towards an affirmative answer to the question above, our discussion below will indicate that 
such optimism is not really justified, and Proposition $\mathrm{C}$ suggests to me that the answer may be negative.

We note two easy consequences of Theorem A.

COROLlaRY A1. Let $G$ be an $\mathfrak{B}$-group. If every proper subgroup of $G$ is almost locally soluble, then $G$ is linear.

This is clear since in Theorem A, the group $C$ is not almost locally soluble. This corollary is one of the steps in the proof of a theorem of Kleidman and Wilson [12], who go on to show that if $G$ is infinite, then $G \cong \operatorname{PSL}(2, k)$ or $\mathrm{Sz}(k)$, for a suitable locally finite field $k$. The approach of this paper was inspired by theirs.

Corollary A2. Let $G$ be a countable $\mathfrak{G}$-group in which, for some prime $p$, the maximal p-subgroups are conjugate. Then $G$ is linear.

This follows because every section of $G$ will have only countably many maximal $p$-subgroups [8], while $C / D$ clearly has $2^{\aleph_{0}}$ of them. This corollary is a special case of a theorem of Kegel [10] and is an important ingredient of his proof. Theorem 2.4 of Kegel [10], on singular $p$-subgroups of finite simple groups, follows from Theorem B below.

Let us now discuss the proof of Theorem A. First, it suffices to deal with the case when $G$ is countable, as every countable set of elements of an $\mathfrak{G}$ group lies in a countable $\mathfrak{G}$-subgroup [11] and every non-linear group has a countable non-linear subgroup. Then $G$ has a Kegel sequence, that is, a sequence $G_{1}<G_{2}<\cdots$ of finite subgroups such that

$$
\bigcup_{i=1}^{\infty} G_{i}=G,
$$

and

(1.2) $G_{i+1}$ contains a maximal normal subgroup $M_{i+1}$ such that $G_{i} \cap M_{i+1}=1 \quad(i \geq 1)$.

For this, see $[11,4.5]$. Applying the classification of finite simple groups to the simple groups $\bar{G}_{i}=G_{i} / M_{i}$, we eventually find that either $G$ is linear, or

(1.3) (after modifying the Kegel sequence if necessary) the $G_{i}$ are either all alternating groups and of unbounded degrees, or all belong to a fixed family of finite simple classical groups over various fields, with unbounded rank parameter.

See [6] for a more detailed discussion. We are concerned with situation (1.3), and Theorem A will be deduced from the following. 
THEOREM B. There exists an integer-valued function $f(n, r)$ with the following properties.

Let $H$ be a finite group of order at most $n$, and suppose that $H \leq S$, where $S$ is either an alternating group of degree at least $f=f(n, r)$, or a finite simple classical group whose natural projective representation has degree at least $f$.

Then there exist subgroups $D \triangleleft C \leq S$ such that

(i) $[H, C] \leq D$,

(ii) $H \cap C \leq D$,

(iii) $C / D \cong \operatorname{Alt}(r)$,

(iv) $D=1$ if $S$ is alternating, and is a p-group of class at most two and exponent dividing $p^{2}$ if $S$ is a classical group over a field of characteristic $p$.

Roughly speaking, a small subgroup of a large alternating or classical group centralizes a large section. This theorem would be more satisfactory if one could always take $D=1$, and at first sight it is tempting to work towards that goal. The next result shows that it is impossible.

Proposition C. Let $p$ be a given prime. Then there exists a finite group $H$ that can be embedded in infinitely many groups $\operatorname{PSL}(n, p)$ as a subgroup with trivial centralizer. If $p \neq 2$ we can take $|H|=2 p^{3}$.

Though this result suggests that our main question has a negative answer, it does not prove it. That would require the embeddings to be induced by embeddings of the projective special linear groups.

The argument of Proposition C yields rather more. In fact, $H$ also embeds in the corresponding groups $\operatorname{SL}(n, p)$ in such a way that the only matrices centralizing it are scalar. Thus, $H$ has modules over $\mathbb{F}_{p}$ that have unbounded dimension, but whose endomorphism rings are $\mathbb{F}_{p}$. These modules are, of course, indecomposable. This may be of independent interest. Of course, there are well known examples of finite $p$-groups having indecomposable modules of unbounded dimension over $\mathbb{F}_{p}$, but the radicals of the endomorphism rings of these modules necessarily have unbounded dimensions also. The above phenomenon seems to be new. A more detailed version of Proposition C is given as Proposition C2 in Section 3; Proposition C1 is a related result.

\section{Proofs}

The proof of the first case of Theorem B, when $S=\operatorname{Alt}(m)$, is virtually the same as an argument in [12]. We reproduce it for the reader's convenience and because it is the pattern for the other cases. 
Proof of Theorem B, Case (i), $S=\operatorname{Alt}(m)$. The number of inequivalent transitive permutation representations of our subgroup $H$ of $S$ is the number of conjugacy classes of subgroups of $H$, say $t=t(H)$. Think of $S$ as acting on $\Omega=\{1,2, \ldots, m\}$. Let $r$ be given. If $m \geq \operatorname{tr}|H|$, then in the action of $H$ on $\Omega$, there will be a set of $r$ equivalent orbits. Thus, we can write $\Omega=\Omega_{1} \cup \cdots \cup \Omega_{r} \cup \Omega^{\prime}$, where $\Omega_{1}, \ldots, \Omega_{r}$ are equivalent $H$-orbits and $\Omega^{\prime}$ is permuted by $H$. Let $K$ be a subgroup of $H$ which is the stabilizer of a point in each $\Omega_{i}$, and let $\omega_{i}$ be a point in $\Omega_{i}$ of which $K$ is the stabilizer. For any $\sigma \in \operatorname{Alt}(r)$, let $f(\sigma)$ be the permutation of $\Omega$ sending $\omega_{i} h$ to $\omega_{i \sigma} h \quad(1 \leq i \leq r, h \in H)$ and fixing $\Omega^{\prime}$ pointwise. Clearly, $f$ embeds $\operatorname{Alt}(r)$ in $\operatorname{Alt}(\Omega)=S$. The image $C$ of $f$ commutes elementwise with $H$ and intersects $H$ trivially, so we may take $D=1$ here.

Before dealing with the other cases, we need to establish our notation for classical groups. It will be consistent with [3], where a brief account of them is given; for more detail, see [4]. In particular, the orthogonal groups over a field $k$ of characteristic 2 arise as follows. If $V$ is a vector space of finite dimension over $k$, then a quadratic form on $V$ is a map $g: V \rightarrow k$, together with a $k$-valued bilinear form $($,$) on V$, such that

$$
g(\lambda x+\mu y)=\lambda^{2} g(x)+\lambda \mu(x, y)+\mu^{2} g(y) \quad(x, y \in V, \lambda, \mu \in k) .
$$

The bilinear form is uniquely determined and necessarily alternating. Let $V_{0}$ be the radical of $($,$) and d=\operatorname{dim} V_{0}$, which is called the defect of $g$. The form $g$ is called non-degenerate if $g(x) \neq 0$ for all $0 \neq x \in V_{0}$. When $k$ is finite, there are the following possibilities. If $\operatorname{dim} V=2 a+1$ is odd, then $d=1$ and there is only one equivalence class of non-degenerate quadratic forms on $V$. The associated isometry group $\Omega(2 a+1, k)$ is isomorphic to $\operatorname{Sp}(2 a, k)$ so from the group-theoretical point of view, odd dimensional orthogonal groups need not be considered. If $\operatorname{dim} V=2 a$, then $d=0$ and there are two equivalence classes of non-degenerate quadratic forms, giving rise to simple groups $P \Omega^{ \pm}(2 a, k)$ as in the odd characteristic case.

We need the following result ("Witt's Theorem").

LEMMA 2.1. Let $V$ be a vector space over a finite field $k$, carrying a nondegenerate alternating, quadratic or hermitian form $f$. If char $k=2$ and $f$ is quadratic, assume further that $\operatorname{dim} V$ is even. Let $U_{1}, U_{2}$ be subspaces of $V$ and $\theta: U_{1} \rightarrow U_{2}$ be an isometry. Then $\theta$ can be extended to an isometry of $V$.

These facts can be found in [4, page 21] ( $f$ hermitian or $f$ quadratic in odd characteristic), [7, page 23] ( $f$ alternating) and [4, page 36] ( $f$ quadratic in characteristic 2). 
Continuing with the proof of the theorem, we now consider

Proof of Theorem B, Case (ii), $S$ is a classical group. Thus $S$ is constructed from a vector space $V$ of finite dimension $m$ over a finite field $k$. We have $S=T / Z$, where $Z$ acts scalarly on $V$ and $T=\operatorname{SL}(V), \operatorname{Sp}(V), \Omega^{ \pm}(V)$ or $\mathrm{SU}(V)$. Except in the first case, $V$ carries a form of the appropriate kind. This will be denoted by $g$ in the case of orthogonal groups in characteristic 2 , and $($,$) otherwise. We have to show that if m$ is sufficiently large compared with $r$ and $|H|$, then $C$ and $D$ can be found with the stated properties. Let $H=L / Z$, where $L \leq T$. We proceed in a number of steps.

(a) If $m>4|H|$, then $L$ leaves invariant a non-zero totally isotropic (respectively totally singular) subspace of $V$.

Recall that if $V$ carries a quadratic form $f$, a subspace $U$ of $V$ is called totally singular (for $f$ ) if $f(u)=0$ for all $u \in U$. In the case $T=\operatorname{SL}(V)$, every subspace is considered totally isotropic.

Proof. We consider the various types separately. In the case $T=\operatorname{SL}(V)$, there is nothing to do. In the other cases, let $v_{1}, \ldots, v_{m}$ be a basis of $V$ and let $\mathbf{H}$ be a transversal to $Z$ in $L$. Then

$$
v_{i} h=\sum_{j=1}^{m} \lambda_{i j}(h) v_{j}, \quad\left(\lambda_{i j}(h) \in k, h \in \mathbf{H}, 1 \leq i \leq m\right) .
$$

Consider the case when $T=\mathrm{Sp}(V)$ or $\Omega^{ \pm}(V)$ in odd characteristic, so that our form $($,$) is alternating or symmetric. Let a_{s t}=\left(v_{s}, v_{t}\right) \quad(1 \leq s$, $t \leq m)$. Then

$$
\left(\sum \sigma_{s} v_{s}, \sum \tau_{t} v_{t}\right)=\sum a_{s t} \sigma_{s} \tau_{t} \quad\left(\sigma_{s}, \tau_{t} \in k\right) .
$$

Let $v \in V$. The space $U$ spanned by all $v h(h \in \mathbf{H})$ is $L$-invariant since $Z$ acts scalarly on $V$. Further, $U$ is totally isotropic if and only if $(v, v h)=0$ for all $h \in \mathbf{H}$; again, this is because $Z$ acts scalarly. Thus, if $v=\sum_{s=1}^{m} \sigma_{s} v_{s}\left(\sigma_{s} \in k\right)$, then $U$ is totally isotropic if and only if, for all $h \in \mathbf{H}$, we have

$$
\begin{aligned}
0 & =(v, v h)=\left(\sum \sigma_{s} v_{s}, \sum_{u, t} \sigma_{u} \lambda_{u t}(h) v_{t}\right) \\
& =\sum_{s, u}\left(\sum_{t} a_{s t} \lambda_{u t}(h)\right) \sigma_{s} \sigma_{u} .
\end{aligned}
$$

This is a system of $|H|$ homogeneous quadratic equations over the finite field $k$ for the $m$ variables $\sigma_{1}, \ldots, \sigma_{m}$. By the theorem of Chevalley and Warning [14, page 5] (where the result is misstated), there is a nontrivial solution provided that the sum of the degrees of the equations is less than the number of variables, that is, if $2|H|<m$. 
Now let $T=S U(V)$, so that (, ) is a non-degenerate hermitian form. We have $|k|=q^{2}$ for some prime power $q$. Let $\lambda \mapsto \bar{\lambda}$ be the unique automorphism of order 2 of $k$, and $k_{0}$ be its fixed field. Let $v_{1}, \ldots, v_{m}$ be as before, and consider a vector $v=\sum_{s=1}^{m} \sigma_{s} v_{s}$ with $\sigma_{s} \in k_{0}$. The space $U$ spanned by the $v h(h \in \mathbf{H})$ is totally isotropic if and only if, for all $h \in \mathbf{H}$,

$$
\begin{aligned}
0 & =(v, v h)=\sum_{s, u}\left(\sum_{t} a_{s t} \overline{\lambda_{u t}(h)}\right) \sigma_{s} \sigma_{u} \\
& =\sum_{s, u} \beta_{s u}(h) \sigma_{s} \sigma_{u}
\end{aligned}
$$

say, with $\beta_{s u} \in k$. Let $1, \omega$ be a $k_{0}$-basis of $k$ and write $\beta_{s u}(h)=$ $\alpha_{s u}(h)+\alpha_{s u}^{\prime}(h) \omega$ with $\alpha_{s u}(h), \alpha_{s u}^{\prime}(h) \in k_{0}$. Then the above yields $2|H|$ homogeneous quadratic equations for $\sigma_{1}, \ldots, \sigma_{m}$, and there will be a nontrivial solution if $m>4|H|$.

There remains the case when $\operatorname{char} k=2, \operatorname{dim} V$ is even, and $V$ carries a non-degenerate quadratic form. With $v=\sum \sigma_{s} v_{s}$ as before, we have

$$
g(v)=\sum \sigma_{s}^{2} g\left(v_{s}\right)+\sum_{s<t} \sigma_{s} \sigma_{t}\left(v_{s}, v_{t}\right)
$$

We now require $g(v h)=0$ and $(v, v h)=0$ for all $h \in \mathbf{H}$. This gives $2|H|$ quadratic conditions, which can be satisfied non-trivially if $m>4|H|$.

(b) Let $d$ be given. If $m \geq 2 d+4|H|$, then $L$ leaves invariant a totally isotropic (respectively totally singular) subspace of $V$ of dimension at least $d$.

Proof. If $T=\operatorname{SL}(V)$, there is again nothing to do. Otherwise, let $U$ be a totally isotropic (respectively totally singular) $L$-invariant subspace of maximal dimension. Then $U \leq U^{\perp}$ (in the case when we have a quadratic form in characteristic 2 , the orthogonal space is taken with respect to the associated symplectic form). We have an induced non-degenerate form on $U^{\perp} / U$, and in its action on this space, $L$ leaves invariant no non-zero totally isotropic (respectively totally singular) subspace. By (a), we have $m-2 \operatorname{dim} U=\operatorname{dim} U^{\perp} / U \leq 4|H|$, whence $2 \operatorname{dim} U \geq m-4|H|$. If $m \geq 2 d+4|H|$, this gives $\operatorname{dim} U \geq d$, as required.

(c) An L-composition series of $V$ contains at most $|H|$ isomorphism types of factors, each of dimension at most $|H|$.

Proof. There is a unique one-dimensional $k Z$-module $W$ such that $V_{z}$ is a direct sum of copies of $W$. If $X$ is any $L$-composition factor of $W$, then $X_{Z}$ is also a direct sum of copies of $W$. Then $\operatorname{Hom}_{Z}\left(X_{Z}, W\right) \neq 0$, and by Nakayama's form of Frobenius Reciprocity [9, V.16.6], $\operatorname{Hom}_{L}\left(X, W^{L}\right) \neq$ 0 . Hence $X$ can be embedded in $W^{L}$, which has dimension $|H|$.

(d) If $Y$ is any $k L$-submodule of $V$ and $S(Y)$ is the socle of $Y$, then $\operatorname{dim} Y \leq|H| \operatorname{dim} S(Y)$. 
Proof. $Y$ is an essential extension of its socle $S(Y)$, and so can be embedded in the injective hull of $S(Y)$. The $k L$-module $W^{L}$ considered in (c) is injective, since $|Z|$ is coprime to char $k$ and for finite groups, induction preserves the property of being injective. Clearly $S(Y)$ is a direct sum of at most $\operatorname{dim} S(Y)$ simple submodules, and each of these can be embedded in the injective module $W^{L}$, and so $S(Y)$ can be embedded in the direct sum of $\operatorname{dim} S(Y)$ copies of $W^{L}$, an injective module of dimension $|H| \operatorname{dim} S(Y)$. By our first remark, $Y$ can also be embedded in this module.

(e) If $m \geq 2 r|H|^{3}+4|H|$, then $V$ contains an L-submodule $U$ which is the direct sum of $r$ isomorphic simple L-submodules and is totally isotropic (respectively totally singular).

Proof. Let $W$ be an $L$-invariant totally isotropic (respectively totally singular) subspace of $V$ of maximal dimension. By (c), if $\operatorname{dim} S(W)>$ $(r-1)|H|^{2}$, then some simple $k L$-module must occur at least $r$ times in $S(W)$. By (b), if $m \geq 2 r|H|^{3}+4|H|$, then $\operatorname{dim} W \geq r|H|^{3}$, whence (d) gives $\operatorname{dim} S(W) \geq r|H|^{2}$. Thus, we may take $U=S(W)$.

(f) Completion of proof. We consider only the case when some nontrivial form is involved. The interpretation for $\operatorname{SL}(V)$ will be clear. We put $f(r, n)=2 r n^{3}+4 n$. By (e), if $m \geq f(r, n)$, then $V$ contains a $k L$ submodule of the form $U=U_{1} \oplus \cdots \oplus U_{r}$, where the $U_{i}$ are copies of a simple $k L$-module, and $U$ is totally isotropic or totally singular, as the case may be. We have an action of $A=\operatorname{Alt}(r)$ on $U$ by permuting the summands; this is trivially an action by isometries of $U$, and can be arranged to commute with the action of $L$. We have $0 \leq U \leq U^{\perp} \leq V$. Write $U^{\perp}=U \oplus W$, and extend the action of $A$ to $U^{\perp}$ by allowing it to act as the identity on $W$. It is easy to see that this is an action by isometries of $U^{\downarrow}$. By Lemma 2.1, the action of each $a \in A$ on $U^{\perp}$ can be extended to an isometry $\rho_{a}$ of $V$. Since $A$ is perfect, we can arrange that $\rho_{a} \in T$. Let $C^{*}=N_{T}(U) \cap C_{T}\left(U^{\perp} / U\right)$ and $D_{1}=C_{C^{*}}(U)$. Then $\rho_{a} \in C^{*}$, and if $C_{1}=\left\langle\rho_{a}: a \in A\right\rangle D_{1}$, then $C_{1} / D_{1} \cong A$ via restriction to $U$. We also have $L \leq N_{T}(U)$, and hence $L \leq N_{T}\left(U^{\perp}\right)$. Therefore $\left[L, C_{1}\right] \leq C_{T}(U) \cap C_{T}\left(U^{\perp} / U\right)=D_{1}$. Also $L \cap C_{1}$ operates trivially on $U$, as $C_{1}$ operates by permuting $U_{1}, \ldots, U_{r}$ while $H$ leaves them invariant, and hence $L \cap C_{1} \leq D_{1}$.

Next note that $D_{1}$ operates trivially on $V / U^{\perp}$. For if $v \in V, u \in U$ and $x \in D_{1}$, then $(v x, u)=\left(v, u x^{-1}\right)=(v, u)$, so $(v x-v, u)=0$ and $v x-v \in U^{\perp}$. Hence $D_{1}$ operates trivially on $V / U^{\perp}, U^{\perp} / U$ and $U$ and so it is a nilpotent group of class at most two and exponent dividing $p^{2}$.

Finally, put $C=C_{1} Z / Z, D=D_{1} Z / Z$ and recall that $H=L / Z$. The required conditions are trivial to verify. 
REMARK. If $p$ does not divide $|H|$, then the subspace $W$ above can be taken to be $L$-invariant.

Deduction of Theorem A. Let $F$ be a given finite subgroup of $G$ and $r_{1}, r_{2}, \ldots$ be a strictly increasing sequence of natural numbers. Let $G_{1}<$ $G_{2}<\cdots$ be a Kegel sequence of $G$. We may assume that $F \leq G_{1}$ and that the simple groups $G_{i}$ are either all alternating and have unbounded degrees, or belong to a fixed family $\operatorname{PSL}(n,-), \operatorname{PSp}(n,-), \ldots$ in which the parameter $n$ is unbounded.

Put $C_{0}=D_{0}=1$ and choose subgroups $D_{t} \triangleleft C_{t}$ for $t>0$ as follows. Having obtained $C_{t}$, choose $k$ such that $C_{t} \leq G_{k}$. For any $s \geq k$, we can apply Theorem B to the subgroup $G_{k} M_{s} / M_{s}$ of the simple group $G_{s} / M_{s}$, and thus, by choosing $s$ large enough, we have subgroups $M_{s} \leq L_{t+1} \triangleleft K_{t+1} \leq$ $G_{s}$ such that $\left[G_{k}, K_{t+1}\right] \leq L_{t+1}, G_{k} M_{s} \cap K_{t+1} \leq L_{t+1}$, and $K_{t+1} / L_{t+1} \cong$ $\operatorname{Alt}\left(r_{t+1}\right)$. Further, $L_{t+1} / M_{s}$ has prime power order. Put $C_{t+1}=C_{t} K_{t+1}$ and $D_{t+1}=D_{t} L_{t+1}$. Finally, set $C=\bigcup_{t=1}^{\infty} C_{t}$ and $D=\bigcup_{t=1}^{\infty} D_{t}$. Clearly $D \triangleleft C$, and $[C, F] \leq D$ as $F \leq G_{1}$. We shall show that

$$
C \cap F \leq D
$$

and

$$
C / D=\operatorname{Dr}_{t=1}^{\infty} K_{t} D / D \cong \operatorname{Dr}_{t=1}^{\infty} K_{t} / L_{t}
$$

We are using Dr to denote direct product here. Clearly $(*)$ follows from $(* *)$, since $C \cap F$ is central modulo $D$. Now if $t<s$, then $\left[K_{t}, K_{s}\right] \leq$ $\left[C_{t}, K_{s}\right] \leq L_{s} \leq D$, and so the groups $K_{t} D / D$ commute elementwise. Since they are non-abelian simple or trivial, $C / D$ is their direct product. Finally, we have $K_{t} D / D \cong K_{t} /\left(K_{t} \cap D\right)$, and we wish to show that $K_{t} \cap D=L_{t}$ if $t \geq 1$. Now

$$
K_{t} \cap D_{t}=K_{t} \cap D_{t-1} L_{t}=\left(K_{t} \cap D_{t-1}\right) L_{t}=L_{t} .
$$

Suppose that for some $s \geq t$, we know that $K_{t} \cap D_{s}=L_{t}$. Then

$$
K_{t} \cap D_{s+1} / L_{t}=K_{t} \cap D_{s+1} / K_{t} \cap D_{s} \cong\left(K_{t} \cap D_{s+1}\right) D_{s} / D_{s},
$$

and

$$
K_{t} \cap D_{s+1} \leq C_{s} \cap D_{s} L_{s+1}=D_{s}\left(C_{s} \cap L_{s+1}\right) .
$$

Now from the way these subgroups are constructed, we have a certain subgroup $G_{k}$ containing $C_{s}$ and a number $j>k$ such that $M_{j} \leq L_{s+1} \triangleleft K_{s+1} \leq$ $G_{j}$. Then $C_{s} \cap M_{j}=1$, and so $C_{s} \cap L_{s+1} \cong\left(C_{s} \cap L_{s+1}\right) M_{j} / M_{j}$, which has prime power order. Thus, $\left(K_{t} \cap D_{s+1}\right) / L_{t}$ is a normal subgroup of prime power order of the non-abelian simple group $K_{t} / L_{t}$, and must therefore be trivial. Therefore $K_{t} \cap D_{s+1}=L_{t}$. It follows that $K_{t} \cap D_{s}=L_{t}$ for all $s \geq t$, 
and hence that $K_{t} \cap D=L_{t}$, as required to complete the proof of Theorem B.

\section{Examples}

In this section, let $U$ denote a vector space of finite or infinite dimension over a field $k$. Let $E$ be the ring of $k$-linear maps of $U$ into itself, which we consider to act on the right. Let $V=U \oplus U$ and $F$ be the ring of $k$-linear maps of $V$ into itself. We think of $V$ as consisting of row vectors of length 2 with entries from $U$, and $F$ as the $2 \times 2$ matrix ring $M_{2}(E)$, acting on $V$ by right multiplication.

LEMMA 3.1. Let $S$ be a subset of $E$ containing the identity map 1, let $C$ be the centralizer ring $C_{E}(S)$ of $S$ in $E$, and let $\xi=\left(\begin{array}{ll}u & v \\ w & x\end{array}\right) \in F$. For $s \in S$, let $\beta(s)=\left(\begin{array}{ll}1 & s \\ 0 & 1\end{array}\right)$. Then the following conditions on $\xi$ are equivalent.

(i) For each $s \in S$, there exists a scalar $\lambda(s) \in k$ such that $\xi \beta(s)=$ $\lambda(s) \boldsymbol{\beta}(s) \xi$.

(ii) $w=0$ and $u=x \in C$.

Proof. Assuming (i), and equating matrix entries, we obtain

$$
\begin{array}{cl}
u=\lambda(s)(u+s w) ; & u s+v=\lambda(s)(v+s x) ; \\
w=\lambda(s) w ; & w s+x=\lambda(s) x .
\end{array}
$$

If $\lambda(s) \neq 1$ for some $s$, we see that $\xi=0$. Otherwise, we obtain $0=s w=$ $w s$ and $u s=s x$. Putting $s=1$ gives $w=0$ and $u=x$, and then, as $u s=s u$ for all $s \in S$, we find that $u \in C$. The converse is clear.

If $k$ has characteristic $p>0$, then the matrices $\beta(s)$ generate an elementary abelian $p$-group $B$ of rank at most $|S|$. The centralizer $C_{F}(B)$ has an ideal $J$ of square zero such that $C_{F}(B) / J \cong C$, and thus any decomposition of the identity into a sum of two non-zero orthogonal idempotents in $C_{F}(B)$ gives one in $C$. Hence if $U$ is $S$-indecomposable, then $V$ is $k B$-indecomposable. Taking $S=\{1, s\}$ for various choices of $s$ allows one to construct a number of examples of indecomposable modules over $\mathbb{F}_{p}$ for an elementary abelian group of order $p^{2}$. This kind of thing is familiar, but we need to proceed a little further.

LEMMA 3.2. Continuing with the same notation, assume that $C=k$. Let $\rho$ be a homomorphism of a group $G$ into $\mathrm{GL}(U)$, and for $g \in G$, let

$$
\rho^{*}(g)=\left(\begin{array}{cc}
1 & 0 \\
0 & \rho(g)
\end{array}\right) \text {. }
$$


Assume that (i) of Lemma 3.1 holds, and also that for each $g \in G$, there exists $\mu(g) \in k$ such that $\xi \rho^{*}(g)=\mu(g) \rho^{*}(g) \xi$. Then $\xi \in k$ provided either

(i) no one-dimensional subspace of $U$ is $\rho(G)$-invariant, or

(ii) $u \neq 0$ and no non-zero vector in $U$ is $\rho(G)$-invariant.

Proof. We have, by Lemma 3.1, that $\xi=\left(\begin{array}{ll}u & v \\ 0 & u\end{array}\right)$ with $u \in k, v \in E$. The equation $\xi \rho^{*}(g)=\mu(g) \rho^{*}(g) \xi$ gives $v \rho(g)=\mu(g) v$, whence, if $y \in U$, $y v \rho(g)=\mu(g) y v$. Hence the subspace spanned by $y v$ is $\rho(G)$-invariant. Under hypothesis (i) we get $y v=0$, and since $y$ is any element of $U$, we deduce that $v=0$. In case (ii) we get $u=\mu(g) u$ for all $g \in G$, whence $\mu(g)=1$ and the above shows that $y v$ is $\rho(G)$-invariant. Hence $y v=0$ and $v=0$ as before.

As a first application of these observations, take $k$ to be a field of odd prime characteristic $p$, and take $S=\left\{1, s_{1}, s_{2}\right\}$, where $s_{1}$ and $s_{2}$ are chosen to make sure that $C=k$. Let $G$ be cyclic of order 2 , generated by $g$, and let $\rho(g)=-1$. Then $H=\langle\rho(G), B\rangle$ is of order $2 p^{3}$, with an elementary abelian normal subgroup of order $p^{3}$ inverted by an involution. If $U$ has finite dimension $n$, we can take $s_{1}$ to be $1+\sum_{i=1}^{n-1} e_{i, i+1}$ where the $e_{i j}$ are the usual elementary matrices, and $s_{2}$ to be the transpose of $s_{1}$. Another possibility is to take $U$ to be the $k$-space with basis $x_{i} \quad(i \in Z)$, with $s_{1}$ as the shift $x_{i} \mapsto x_{i+1}$, and $s_{2}$ interchanging $x_{1}$ and $x_{2}$ and fixing the other basis elements. Then $\left\langle s_{1}, s_{2}\right\rangle$ contains every finitary permutation of the basis elements, and so $C=k$. Thus we obtain the following.

Proposition C1. Let $p$ be an odd prime and $k$ be a field of characteristic $p$. Then there is a finite group $H$ of order $2 p^{3}$ and there are $k H$ modules $V_{1}, V_{2}, \ldots, V$ such that $\operatorname{dim} V_{i}=2 i, \operatorname{dim} V$ is countably infinite, and $\mathrm{End}_{k H} V_{i}=\mathrm{End}_{k H} V=k$. In particular, these modules are indecomposable. Furthermore, the centralizer of the image of $H$ in the corresponding projective group is trivial.

More generally, let $k$ be any field of characteristic $p>0$, and let $G$ be any nontrivial finite group such that $O_{p}(G)=1$. It is easy to see that the kernels of the nontrivial irreducible representations of $G$ intersect trivially, and so $G$ has a faithful representation $\sigma$ over $k$ leaving no nonzero vector invariant. Let $\sigma$ have dimension $d$ and $m$ be any multiple of $d$. Let $U$ be an $m$-dimensional vector space over $k$ and let $s_{1}=1+\sum_{i=1}^{m-1} e_{i, i+1}$ and $s_{2}$ be the transpose of $s_{1}$ as before. Let $\rho$ be the direct sum of $m / d$ copies of $\sigma$, viewed as a representation of $G$ on $U$. Then by Lemma 3.2, the centralizer of the image in $\operatorname{PGL}(V)$ of $H=\left\langle\rho^{*}(G), B\right\rangle$ is trivial. This image is isomorphic to $H$, and there is an exact sequence $1 \rightarrow \bar{B} \rightarrow H \rightarrow G \rightarrow 1$, 
where $\bar{B}$ is the normal closure of $B$ under $\rho^{*}(G)$. Clearly $|B|$ divides $p^{3|G|}$, and so the same group occurs as $H$ infinitely often as $m$ increases. Since the determinants of the matrices $\sigma(g)(g \in G)$ are $|G|$ th roots of 1 , we can arrange that $H \leq \mathrm{SL}(V)$ for infinitely many values of $m$. This gives the following.

Proposition C2. Let $p$ be any prime and $k$ a field of characteristic $p$. Let $G$ be any nontrivial group such that $O_{p}(G)=1$. Then there exists $a$ finite group $H$ of the form $A \rtimes G$, where $A$ is an elementary abelian normal p-subgroup of $G$, such that $H$ can be embedded in infinitely many groups $\operatorname{PSL}(n, k)$ as a subgroup with trivial centralizer.

\section{References}

[1] V. V. Belyaev, Locally finite Chevalley groups, Studies in Group Theory, (Acad. of Sciences of the U.S.S.R., Urals Scientific Centre, 1984).

[2] A. V. Borovik, 'Embeddings of finite Chevalley groups and periodic linear groups', Sibirsk. Mat. Zh. 24 (1983), 26-35 (Russian) =Siberian Math. J. 24 (1983), 843-851.

[3] R. W. Carter, Simple groups of Lie type (Wiley, New York, 1972).

[4] J. Dieudonné, La géométrie des groupes classiques, Ergebnisse der Math. und ihrer Grenzgebiete 5 (Springer-Verlag, Berlin, 1955).

[5] J. I. Hall, Infinite alternating groups as finitary linear transformation groups, J. Algebra 119 (1988), 337-359.

[6] B. Hartley and M. Kuzucuoglu, 'Centralizers of elements in locally finite simple groups', Proc. London Math. Soc., to appear.

[7] B. Hartley and G. Shute, 'Monomorphisms and direct limits of finite groups of Lie type', Quart. J. Math. Oxford (2) 33 (1982), 309-323.

[8] B. Hartley, 'Sylow subgroups of locally finite groups', Proc. London Math. Soc. (3) 23 (1971), 159-192.

[9] B. Huppert, Endliche Gruppen I (Springer-Verlag, Berlin, 1967).

[10] O., H. Kegel, 'Four lectures on Sylow theory in locally finite groups', Group theory (Proc. Singapore Group Theory Conference) (de Gruyter, Berlin, 1989).

[11] O. H. Kegel and B. A. F. Wehrfritz, Locally finite groups (North-Holland, Amsterdam, 1973).

[12] P. B. Kleidman and R. A. Wilson, 'A characterization of some locally finite groups of Lie type', Arch. Math. (Basel) 48 (1987), 10-14.

[13] R. E. Phillips, 'The structure of groups of finitary transformations', J. Algebra 119 (1988), 400-448.

[14] J. P. Serre, $A$ course in arithmetic (Graduate Texts in Math. No. 7, Springer-Verlag, Berlin, 1973).

[15] S. Thomas, 'The classification of the simple periodic linear groups', Arch. Math. 41 (1983), 103-116.

University of Manchester

Manchester M13 9PL

England 